\title{
UTILIZACIÓN DE IONES COBRE EN SOLUCIÓN PARA LA PRESERVACIÓN A BORDO DE ANCHOVETA (Engraulis ringens J.), DESTINADA A LA ELABORACIÓN DE HARINA DE PESCADO
}

\section{USE OF COPPER IONS IN SOLUTION FOR ONBOARD PRESERVATION OF ANCHOVIES (Engraulis ringens J.) DESTINED FOR FISHMEAL PRODUCTION}

\author{
David Roldán Acero ${ }^{1}$, Juan Juscamaita Morales ${ }^{1}$, Guillermo Quevedo Neira ${ }^{1}$ y Maria Vásquez Huamán ${ }^{1}$
}

\begin{abstract}
Resumen
Se evaluó el uso del ión cobre en solución para preservar a bordo la anchoveta (Engraulis ringens J.), destinada a la producción de harina de pescado. Los resultados indicaron que la utilización de $7 \mathrm{mg} / \mathrm{L}$ de iones cobre en solución fue eficiente, hasta las 30 horas de almacenamiento en condiciones ambientales, para preservar la anchoveta destinada a la elaboración de harina de pescado de buena calidad. Así mismo, dicha condición de almacenamiento posibilitó la reducción de su contenido de nitrógeno básico volátil total (TVB-N) desde $35.61 \mathrm{mg} / 100 \mathrm{~g}$ hasta $20.04 \mathrm{mg} / 100 \mathrm{~g}$. De igual forma la disminución de la carga microbiana hasta valores de $9.7 \times 10^{4}$ ufc/g permitió mantener el calificativo de pescado fresco por 15 horas de almacenamiento. La variación del valor peróxido en el aceite de anchoveta almacenada demostró que el ión cobre acelera los cambios oxidativos a partir de las 15 horas de almacenamiento.
\end{abstract}

Palabras clave: anchoveta, preservación a bordo, iones cobre.

\begin{abstract}
This research aided in assessing the use of copper ions in solution to preserve onboard anchovies (Engraulis ringens J.), destined for fishmeal production. The results indicated that the use of $7 \mathrm{mg} / \mathrm{L}$ of copper ions in solution was efficient, for up to 30 hours of storage at ambient conditions, in preserving anchovies used for high quality fishmeal production. Also, such storage conditions reduced the content of total volatile basic nitrogen (TVB-N) from $35.61 \mathrm{mg} / 100 \mathrm{~g}$ to $20.04 \mathrm{mg} / 100 \mathrm{~g}$. Similarly, the reduction of microbial loads to levels of $9.7 \times 10^{4} \mathrm{cfu} / \mathrm{g}$ allowed maintaining its qualification as fresh fish for 15 hours of storage. Change in the peroxide value in oils obtained from anchovies stored in the above conditions showed that copper ions accelerate oxidative changes from 15 hours of storage onwards.
\end{abstract}

Key words: anchovy, onboard preservation, copper ions.

\section{Introducción.}

Uno de los principales problemas en la producción de harina de pescado especial (Prime o Superprime), es mantener el grado de frescura del pescado. La anchoveta, principal materia prima destinada a la producción de harina de pescado, sufre alteraciones físicas, químicas y microbiológicas desde su captura. Por esta razón, la industria pesquera se ha preocupado por conservar la calidad de la materia prima que ingresa a las plantas de proceso, ya que este factor tiene una influencia directa en el precio de harina y aceite de pescado y por ende en el nivel de ingresos por venta de estos productos.

La anchoveta (Engraulis ringens J.) es un pequeño pelágico de crecimiento rápido y estacional, distribuido en grandes cardúmenes en el mar, desde Punta Aguja (Perú) hasta Talcahuano (Chile). Esta especie es capturada por embarcaciones con red de cerco, en Chimbote, Huarmey, Supe, Huacho, Callao, Pisco e Ilo (IMARPE - ITP, 1996). La anchoveta es una especie sumamente delicada por su tamaño, por la fragilidad de su textura, por las fluctuaciones en el contenido graso a través de las estaciones, por la elevada insaturación de la grasa, etc. Por lo que son necesarios estudios meticulosos que permitan mantener su calidad (Ramírez, 1979).

El grado de alteración del pescado juega un papel importante en el funcionamiento de la planta de proceso, además de su influencia decisiva en la calidad de los productos finales, harina y aceite. Román (1968) menciona que existen diversos métodos para preservar el pescado utilizando coagulantes, preservantes o inhibidores bacterianos de los cuales muy pocos han sido permitidos por sus efectos tóxicos y altos costos.

Según Román (1968) un preservante de pescado debe cumplir los siguientes requisitos: 
- Impedir la proliferación de las bacterias y disminuir la acción enzimática.

- Estar permitido para el consumo humano o eliminarse durante el procesamiento.

- No aumentar mayormente el costo

- No se debe descomponer en compuestos tóxicos durante el procesamiento del pescado o afectar la calidad de la harina.

El grado de frescura del pescado en las plantas harineras se determina según el método sensorial y método químico como la determinación de TVB-N (nitrógeno básico volátil total) que es una medida del grado de descomposición que tiene el pescado y mide principalmente la degradación proteica, por destrucción de aminoácidos y formación consecuente de amoniaco y aminas biogénicas. Según Grados (1996), los valores aceptados para una buena materia prima, oscilan entre 30 a 50 mg/100 g de muestra.

Las empresas pesqueras utilizan rangos de TVB-N en el pescado para determinar la calidad de la harina. La Tabla 1 reporta las cantidades de TVB-N medidos en la descarga de pescado y las calidades de harinas de pescado, elaborados por la empresa Pesquera Capricornio S.A.

Tabla 1: TVB-N (mg /100 g) en pescado según calidad de la harina producida.

\begin{tabular}{cl}
\hline TVB-N & Calidad harina \\
\hline$<30$ & Super Prime A \\
$30-40$ & Prime A \\
$40-50$ & Estándar C \\
$>50$ & Estándar D \\
\hline $\begin{array}{l}\text { Fuente: Pesquera } \\
\text { (2003) }\end{array}$ & Capricornio S.A. \\
\end{tabular}

El cobre es un elemento esencial para los seres humanos y para una gran variedad de especies animales, entre ellos los peces. Muchos autores lo consideran metal traza o micronutriente de gran importancia, porque se une selectivamente a un gran número de enzimas y de proteínas, cumpliendo un papel fundamental en la actividad de oxido-reducción de las enzimas; también está implicado en la respiración celular, en la defensa contra los radicales libres responsables del envejecimiento celular, en funciones de neurotransmisión, es responsable de la biosíntesis en los tejidos, participa en la síntesis de la hemoglobina y tiene un rol en el mecanismo de la transmisión de los genes. Así también tiene un efecto bactericida y bacteriostático (Sequi, 2002).

Investigaciones realizadas por Faúndez y Figueroa (1999) demostraron que las superficies de cobre poseen actividad antibacteriana sobre Salmonella enteritica y Campylobacter jejuni, lo que no se observó al utilizar superficies de acero inoxidable y polímeros.
El cobre ha sido referido mayormente por sus efectos tóxicos; sin embargo es importante resaltar su poder bactericida. Sequi (2002), menciona que el efecto del cobre es bactericida y bacteriostático. Por ello el objetivo de la presente investigación fue evaluar el uso del ión cobre en solución para la conservación de anchoveta (Engraulis ringens J.) a bordo, destinada a la producción de harina de pescado.

\section{Materiales y métodos.}

El trabajo de investigación fue realizado en las instalaciones de la Universidad Nacional Agraria La Molina (UNALM). La anchoveta utilizada fue proporcionada por la empresa Pesquera Capricornio S.A. y recepcionada en recipientes de plástico de 25 $\mathrm{kg}$ de capacidad; posteriormente fueron trasladadas a los laboratorios para los análisis respectivos. Se utilizó una solución de $100 \mathrm{mg} / \mathrm{l}$ de iones de cobre, obtenida por electro deposición en el Laboratorio de Biorremediación de la UNALM.

El análisis físico y sensorial de la anchoveta se realizó sobre una muestra de 50 unidades tomadas al azar, utilizando la tabla de Wittfogel (Ludortf, 1963). La cantidad de sólidos totales en la sanguaza residual se determinó utilizando la metodología propuesta por el Institute Pacific Thecnologists (1972) citado por Rodríguez (2002). Las muestras de anchoveta entera obtenidas al azar fueron molidas y homogenizadas antes de los análisis realizados. El análisis químico proximal se realizó según el procedimiento recomendado por Nagakura (1972). La prueba microbiológica utilizada fue el contaje de aerobios mesófilos viables según procedimiento de Ingram et al. (1983). La determinación de nitrógeno básico volátil total (TVB-N) se realizó según el método de microdifusión de Conway (1950) y el valor peróxido, según el método de Lea modificado (1952), recomendados por Pearson (1976); previamente se realizó la extracción de lípidos de acuerdo a la técnica de Bligh y Dyer (1959). Adicionalmente se determinó el contenido de cobre por espectrofotometría de absorción atómica, según AOAC (2000).

El análisis estadístico de los resultados se realizó según un diseño experimental completamente aleatorio con arreglo factorial, a excepción del análisis del valor peróxido donde se utilizó un diseño completamente aleatorio simple. Para la comparación de medias se utilizó la prueba de Duncan (Conover, 1995).

La investigación fue realizada en dos etapas experimentales. Previamente se instruyó al personal de la embarcación encargada de proporcionar la anchoveta sobre la forma de recolectar las muestras para el estudio y se les solicitó tomar nota de la zona de captura, la hora en que se realizó la cala, la hora de llegada a puerto, el tipo de aparejo utilizado y el tiempo de faena. 
La primera etapa experimental tuvo como objetivo evaluar el efecto de los iones de cobre en solución como preservante de anchoveta y determinar una concentración de iones de cobre en la solución que permita mayor vida útil de la anchoveta. Se utilizaron tres concentraciones diferentes de soluciones de cobre: Variable $\mathrm{V}_{\mathrm{a}}(3 \mathrm{mg} \mathrm{Cu} / \mathrm{L})$, Variable $\mathrm{V}_{\mathrm{b}}(5 \mathrm{mg} \mathrm{Cu} / \mathrm{L})$, Variable $\mathrm{V}_{\mathrm{c}}(7 \mathrm{mg} \mathrm{Cu} / \mathrm{L})$ y agua de mar como testigo (Variable $\mathrm{T}_{1}$ ).

La segunda etapa experimental permitió determinar los cambios sensoriales, químicos y microbiológicos de la anchoveta almacenada en la solución de iones de cobre obtenida de la mejor variable de la primera etapa experimental y determinar el tiempo de conservación de la anchoveta que permita elaborar una harina de buena calidad. Se consideró como muestra testigo el agua de mar (Variable $\left.\mathrm{T}_{2}\right)$.

\section{Resultados y discusión. \\ Materia prima}

La longitud y peso promedio de la anchoveta utilizada fueron $\begin{array}{lllll}13.35 & \mathrm{~cm} & \mathrm{y} & 24.82 & \mathrm{~g}\end{array}$ respectivamente. Según estos resultados, la anchoveta se encontraría en su etapa adulta ya que se ubicó dentro del rango de los tamaños empleados para la pesca comercial según Jordan y Chirinos (1965). Su estadio sexual, según los grupos de Naier (Laevastu, 1971), correspondería al pre-desove lo que coincide con la época de captura (mayo-junio).

El análisis químico proximal de la muestra de anchoveta reportó la siguiente composición: $19.30 \%$ de proteína, $10.00 \%$ de grasa, $68.60 \%$ de humedad y $1.71 \%$ de ceniza; valores similares a los reportados por IMARPE/ITP (1996) y Ayala et al. (2001). Shirasaka y Arakaki (1975) y Román (1968) mencionan que los componentes químicos del pescado varían según la estación del año en que se captura, edad, madurez sexual, grado de nutrición, región del cuerpo, etc.

\section{Faenas de pesca}

La pesca de la anchoveta (entre mayo y junio) se realizó utilizando redes de cerco, aproximadamente a $12^{\circ} \mathrm{LS}$ y $77^{\circ} \mathrm{LW}$ de la costa. La embarcación utilizada no contó con sistema de refrigeración para la conservación de pescado a bordo. Luego de preparadas las diferentes variables, fueron almacenadas en condiciones ambientales $\left(15^{\circ} \mathrm{C}\right)$ y transportadas a la planta de procesamiento de harina de pescado de la empresa Pesquera Capricornio S.A. (Callao).
Para ambas etapas experimentales, las muestras de anchoveta fueron obtenidas de la primera cala de pesca y almacenadas en baldes de $25 \mathrm{~kg}$. La relación anchoveta y solución de iones de cobre fue 2:1.

Primera etapa experimental

Los análisis sensoriales de la anchoveta se realizaron después de 13 horas de su captura. Inicialmente, la anchoveta de las variables $\mathrm{T}_{1}$ y $\mathrm{V}_{\mathrm{a}}$ obtuvieron el calificativo de Regular, mientras que las muestras de las variables $\mathrm{V}_{\mathrm{b}}$ y $\mathrm{V}_{\mathrm{c}}$ alcanzaron el calificativo de Bueno. Según las características presentadas en las muestras de anchoveta de las variables, se puede mencionar que los indicadores sensoriales de deterioro de la anchoveta se presentaron de forma inversa a las concentraciones de cobre en solución. La actividad bacteriana fue atenuada por la

Figura 1. Contenido de sólidos totales en sanguaza residual según variables en estudio durante el almacenamiento.

presencia de iones cobre, apreciándose en menor intensidad las características de deterioro en la anchoveta de la variable $\mathrm{V}_{\mathrm{c}}$. Los resultados de la determinación de sólidos en la sanguaza residual de las variables estudiadas, se muestran en la Figura 1.

Se encontraron diferencias significativas $(\mathrm{P}<0.05)$ en el contenido de sólidos de las muestras de sanguaza entre las variables $T_{1}$ y $V_{c}$ siendo mayor en la variable $\mathrm{T}_{1}$. Esta diferencia sería propiciada por la presencia de cobre a $7 \mathrm{mg} \mathrm{Cu} / \mathrm{L}$ en solución para la muestra de la variable $\mathrm{V}_{\mathrm{c}}$ dado que los contenidos de sólidos totales en la sanguaza se redujeron. Es posible que el cobre estuviera actuando sobre las bacterias generadoras de enzimas proteolíticas que participan en la autolisis del pescado, retardando con ello la formación de sólidos en la sanguaza.

La prueba de Duncan realizada sobre los contenidos de nitrógeno básico volátil total (TVB-N), indicó diferencias significativas $(\mathrm{P}<0.05)$ en el 
contenido de TVB-N entre las muestras de anchoveta de las variables en estudio. Los valores más altos (158.59 mg/100 g) se presentaron en la muestra de anchoveta de la variable $\mathrm{T}_{1} \mathrm{y}$ los más bajos (1.95 $\mathrm{mg} / 100 \mathrm{~g}$ ) en la muestra de anchoveta de la variable $\mathrm{V}_{\mathrm{c}}$ en las 53 horas de almacenamiento. Los resultados se muestran en la Figura 2. almacenamiento en condiciones ambientales, la calificación alcanzada en ambas muestras fue de muy bueno.

En las unidades de anchoveta de la variable $\mathrm{T}_{2}$, el olor fue una de las características que fue cambiando con las horas de almacenamiento; a las 15 horas aproximadamente se pudo percibir olores ligeramente ácidos que fueron indicadores de principios de deterioro obteniendo por ello el calificativo de regular mientras que en las muestras de la variable $\mathrm{V}_{\mathrm{c}}$ el olor no fue tan fuerte obteniendo el calificativo de bueno. Este resultado confirmaría que el uso de iones cobre en solución proporciona una mejor conservación de la anchoveta, pescado que con 15 horas de almacenamiento al medio ambiente permitiría la elaboración de una harina de buena calidad.

De forma similar a la primera etapa experimental se observó una ligera coloración amarillenta en la superficie de las unidades de anchoveta en ambas muestras,

Grados (1996), refiere un rango de TVB-N entre 30 a $50 \mathrm{mg} / 100 \mathrm{~g}$ de muestra para ser aceptada como una buena materia prima destinada a la elaboración de harina de pescado, valor que se considerará referente para el presente trabajo.

Comparando los resultados obtenidos de TVB-N de las muestras de anchoveta estudiadas con las calidades de harina de pescado según el Tabla 1, a las 33 horas las muestras de anchoveta de las variables $T_{1}$, $\mathrm{V}_{\mathrm{a}}$ y $\mathrm{V}_{\mathrm{b}}$ fueron consideradas como materia prima adecuada para hacer una harina de pescado tipo Prime A, mientras que la muestra de anchoveta de la variable $\mathrm{V}_{\mathrm{c}}$, a la misma hora de almacenamiento, sería destinada para harina de pescado tipo Superprime A.

De acuerdo a los resultados obtenidos, se eligió la variable $\mathrm{V}_{\mathrm{c}}$ que contenía la concentración de $7 \mathrm{mg}$ $\mathrm{Cu} / \mathrm{L}$ en solución, para ser utilizada en la segunda parte experimental del estudio.

Segunda etapa experimental

Los resultados de la evaluación sensorial y del contenido de TVB-N de la anchoveta de la variable $\mathrm{V}_{\mathrm{c}}$ (mejor variable del primer experimento) y $\mathrm{T}_{2}$ (agua de mar) se reportan en la Figura 3.

El análisis sensorial de la anchoveta de las variables $\mathrm{V}_{\mathrm{c}} \mathrm{y} \mathrm{T}_{2}$, se realizó después de 7 horas de

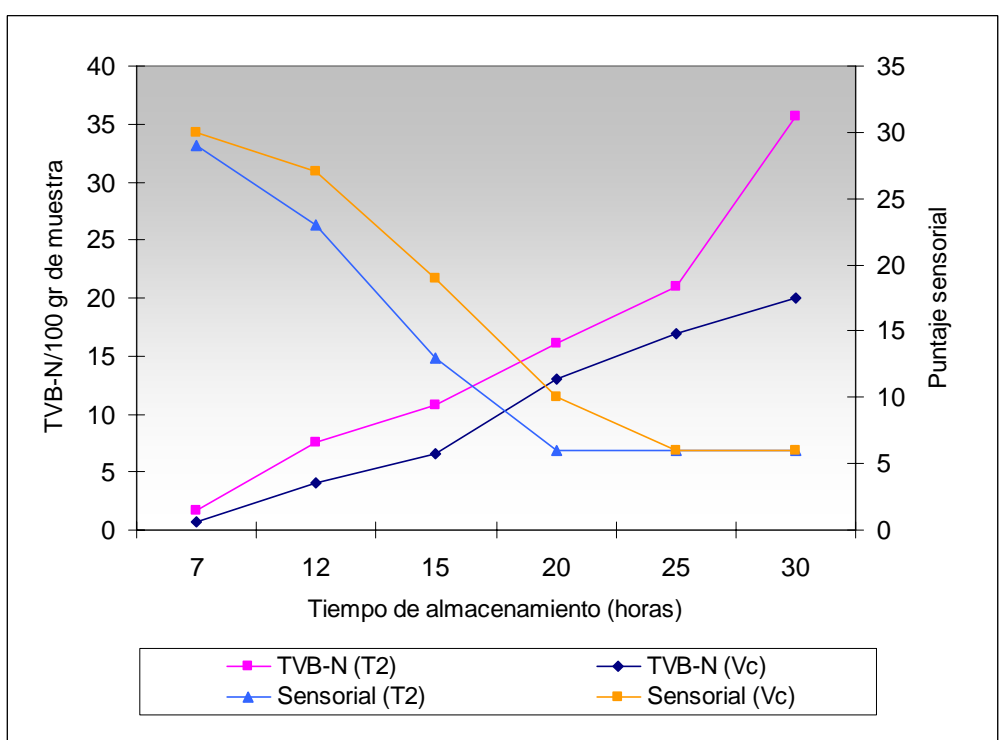

Figura 3. Contenido de TVB-N de la anchoveta almacenada en solución de $7 \mathrm{mg} \mathrm{Cu} / \mathrm{L}$. 
Por otro lado considerando los rangos de TVB-N en la materia prima destinada para cada tipo de harina, recomendada por Pesquera Capricornio S.A. (Tabla 1), la muestra de anchoveta de la variable $V_{c}$, con un contenido de $20.04 \mathrm{mg} / 100 \mathrm{~g}$ hasta las 30 horas de almacenamiento, sería adecuada para elaborar harina de pescado tipo superprime. Sin embargo, la muestra de anchoveta de la variable $\mathrm{T}_{2}$ con un valor de 21,02 mg /100 g estaría considerada como materia prima para este tipo de harina hasta las 25 horas de almacenamiento.

Respecto al análisis microbiológico, el crecimiento de microorganismos psicrófilos y mesófilos en las muestras de anchoveta durante el almacenamiento fueron más elevados en las muestras de la variable $\mathrm{T}_{2}$ que en las de la variable $\mathrm{V}_{\mathrm{c}}$, ello confirmaría que la presencia del cobre estaría inhibiendo los microorganismos presentes en la anchoveta. Estadísticamente según la prueba de Duncan se encontraron diferencias significativas $(\mathrm{P}<0.05)$ entre los recuentos microbianos de psicrófilos y mesófilos de las muestras $\mathrm{T}_{2}$ y $\mathrm{V}_{\mathrm{c}}$ En las Figuras 4 y 5 se muestran los resultados obtenidos.

Tarr (1946), mencionado por Pizardi (1975), estudió la relación entre la frescura del pescado y el contenido de gérmenes viables totales, determinando las siguientes comparaciones: $10^{5} \mathrm{ufc} / \mathrm{g}$ (fresco), $10^{5}-10^{6} \mathrm{ufc} / \mathrm{g}$ (principios de deterioro), mayor a $1.5 \times 10^{6}$ ufc/g (en deterioro). Según la comparación anterior, la anchoveta de la variable $\mathrm{V}_{\mathrm{c}}$ sería considerada como pescado fresco aproximadamente hasta las 15 horas de

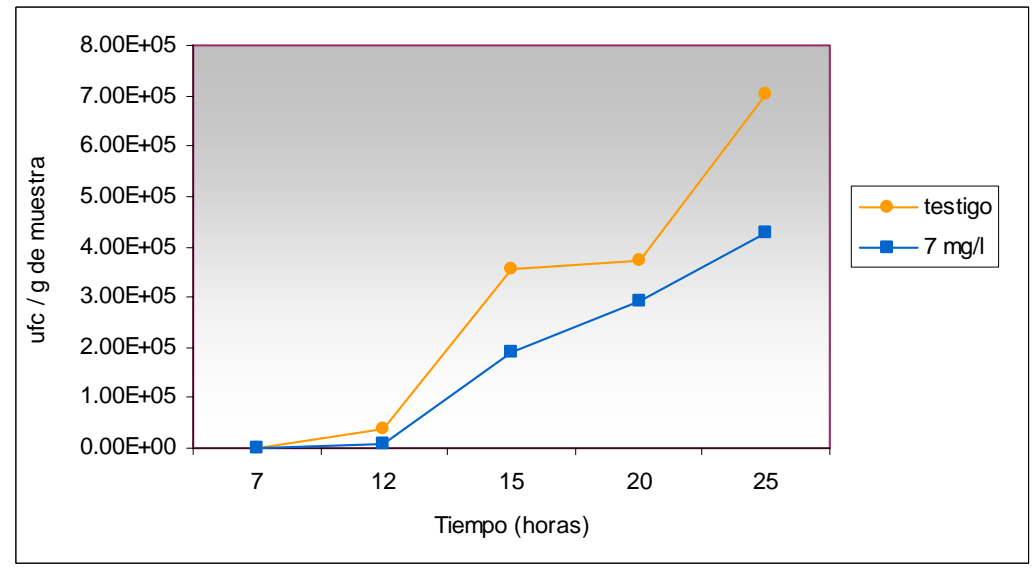

Figura 5. Numeración de microorganismos aerobios mesófilos en músculo de anchoveta conservada en solución de $7 \mathrm{mg} \mathrm{Cu} / \mathrm{L}\left(\mathrm{V}_{\mathrm{c}}\right)$ y Testigo $\left(\mathrm{T}_{2}\right)$. $\left(\mathrm{T}_{2}\right)$. insaturados. almacenamiento y podría ser destinado a la elaboración de harina tipo superprime o prime, mientras que la anchoveta de la variable testigo estaría en principios de deterioro a esa hora de almacenamiento y sería orientada a la elaboración de harina estándar o de calidad inferior.

Los resultados del valor peróxido en los análisis estadísticos según la prueba de Duncan no presentaron diferencias significativas entre las variables $(\mathrm{P}<0.05)$. La evolución del valor peróxido en ambas variables se incrementó rápidamente durante las 30 horas de almacenamiento. A las 20 horas la muestra de la variable $\mathrm{V}_{\mathrm{c}}$ alcanzó el mayor valor peróxido con

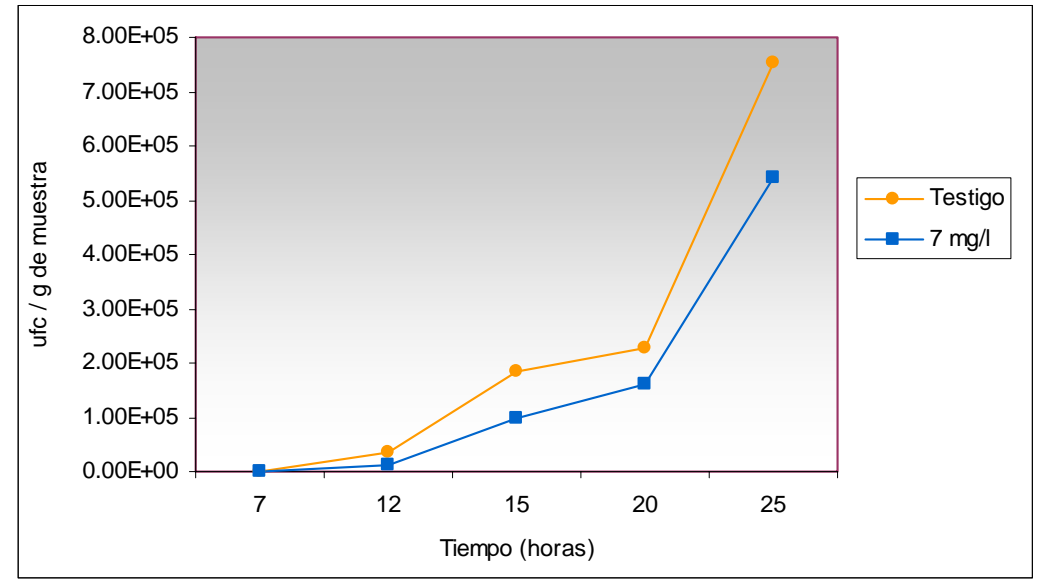

Figura 4. Numeración de microorganismos psicrófilos en músculo de anchoveta conservada en solución de $7 \mathrm{mg} \mathrm{Cu} / \mathrm{L}\left(\mathrm{V}_{\mathrm{c}}\right)$ y Testigo

102.54 meq $\mathrm{O}_{2} / \mathrm{kg}$, a este tiempo el testigo presentó $35.81 \mathrm{meq} \mathrm{O}_{2} / \mathrm{kg}$. Al parecer la presencia de iones cobre en solución participaron como promotores de oxidación, al respecto Ruiter (1999) menciona que las trazas de cobre, hierro y otros metales actúan como catalizadoras en la oxidación de los ácidos grasos

Ricque et al. (1996) reporta sobre la adición de aceites en la alimentación de camarones que los aceites medianamente oxidados con 50 meq $\mathrm{O}_{2} / \mathrm{kg}$ no reportando efectos negativos $\mathrm{y}$ aceites altamente oxidados con $100 \mathrm{meq}$ $\mathrm{O}_{2} / \mathrm{kg}$ no presentando toxicidad significativa, sin embargo disminuyó ligeramente el crecimiento y el consumo de alimento. En la Figura 6 se grafican los resultados obtenidos.

La concentración de cobre residual en la muestra de la variable $\mathrm{V}_{\mathrm{c}}$, a las 26 horas de almacenamiento, fue de 2.9 ppm dicho valor fue 8.69 \% por encima de los reportados por el IMARPE- 


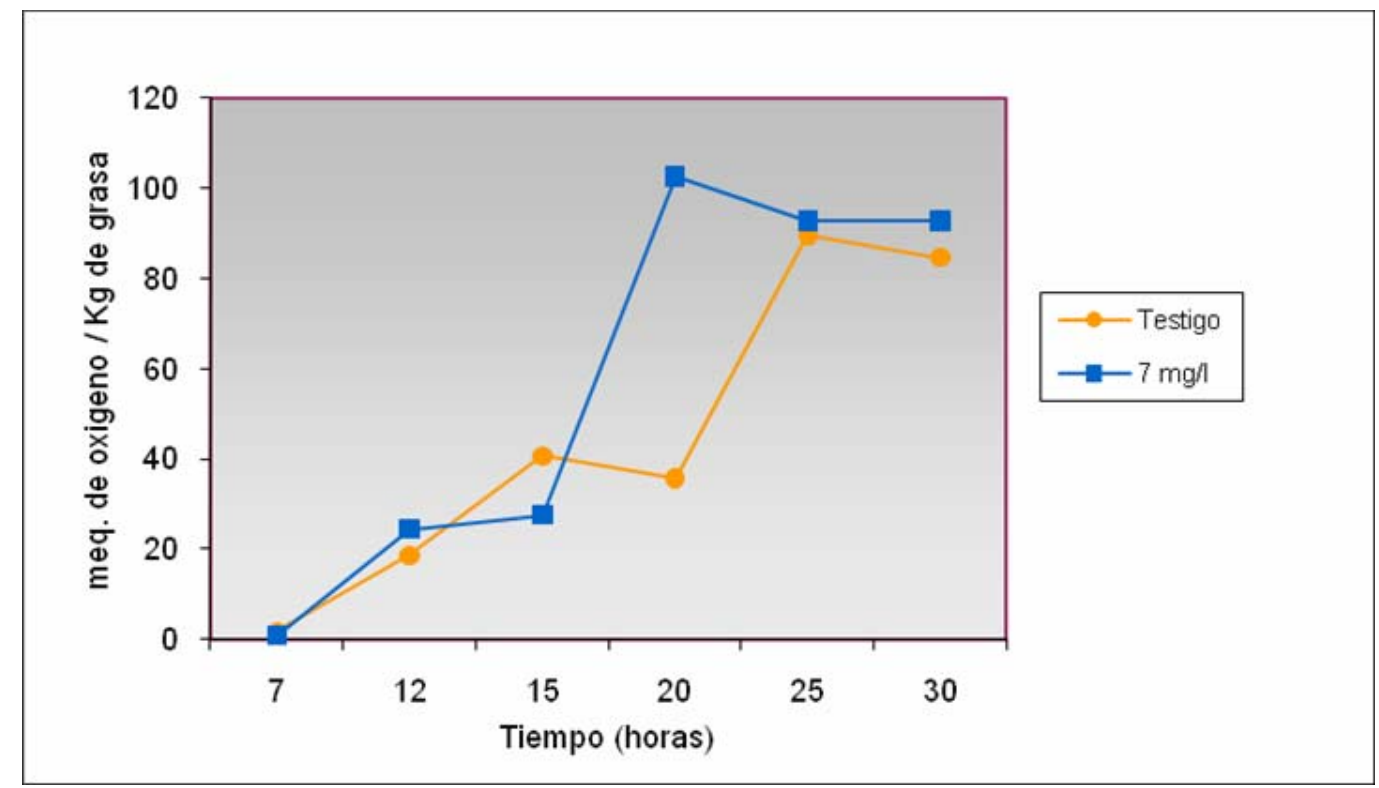

Figura 6. Variación de valor peróxido en la anchoveta en solución de 7 mg Cu/L y Testigo.

ITP (1996) para la anchoveta fresca (2.1 ppm), es necesario continuar con los estudios de este remanente debido a que puede influir en la oxidación de la grasa de las harinas producidas.

Morales et al. (1999) realizaron estudios sobre la determinación de residuos de cobre en carne de pollo y cerdo procesadas experimentalmente sobre superficies de este metal poniendo en evidencia que la concentración de cobre aumentó con el tiempo, alcanzando $2.5 \mathrm{mg}$ de $\mathrm{Cu} / 100 \mathrm{~g}$. de carne a los 50 minutos de exposición; indicaron además que dichos valores no sobrepasan los niveles de ingesta diaria admisible para este nutriente según la FDA y CODEX (2 a 3 mg. de cobre diarios). Por lo anterior, el uso de iones cobre en solución se presenta como una alternativa en la preservación de la anchoveta a bordo destinada a la producción de harina de buena calidad.

\section{Conclusiones.}

Bajo las condiciones experimentales, las conclusiones a las que se llegaron en el presente trabajo de investigación fueron las siguientes:

1 La utilización de 7 ppm de iones de cobre en solución permitió la conservación de la anchoveta (Engraulis ringens J.) destinada a la producción de harina de pescado de buena calidad, hasta las 30 horas de almacenamiento.

2 La conservación de la anchoveta en solución de iones de cobre a $7 \mathrm{mg} \mathrm{Cu} / \mathrm{L}$ redujo, a las 30 horas de almacenamiento, el contenido de TVB-N desde $35.61 \mathrm{mg} / 100 \mathrm{~g}$ hasta $20.04 \mathrm{mg}$ /100g.

3 La utilización de $7 \mathrm{mg} / \mathrm{L}$ de ión cobre en solución permitió disminuir la flora microbiana de la anchoveta hasta $9.7 \times 10^{4}$ ufc/g a las 15 horas aproximadamente de almacenamiento $\mathrm{y}$ se comprobó el efecto bactericida del cobre.

4 La variación del valor peróxido demostró que el ión cobre acelera los cambios oxidativos de la anchoveta a partir de las 15 horas de almacenamiento en condiciones ambientales.

\section{Agradecimientos.}

Nuestro sincero agradecimiento a la Empresa Pesquera Capricornio S.A. y en especial al Superintendente de Planta, Ing. Pedro Paredes, por las facilidades brindadas en la recolección, almacenamiento y trasporte de las muestras de anchoveta.

\section{Literatura citada.}

AOAC. 2000. Official Methods of Analysis. Association of Official Analytical Chemist. $17^{\text {Th }}$ Edition. Meryland. EUA.

Ayala M., Salas A., Carvajal M., Placido M. \& AlbrechtRuíz M. 2001. Patrón de Deterioro de anchoveta peruana (Engraulis ringens J.) almacenada a temperatura de refrigeración. Asociación de Licenciados en Ciencia y Tecnología de los Alimentos de Galicia. 3(3): 161-168.

Bligh E.G. \& Dyer W.J. 1959. A Rapid Method of Total Lipids Extraction and Purification. Canadian Journal of Biochemistry and Physiology. 37 (8).

Conover W.J. 1995. Practical Nonparametric Statistics. Ed. John Wiley \& Sons. Second Edition. New York.

Conway J. 1950. Microdiffusion Analysis and Volumetric Error. Grosby Lonkwood and Son Ltd. London.

Faundez G. \& Figueroa G. (1999) "Cooper Surfaces Inhibit Salmonella Enteritica and Campylobacter jejuni in Food Processing” Unit of Microbiology, INTA, University of Chile. 
Grados L. 1996. Análisis de Riesgos y Puntos de Control Crítico (HACCP) y su Aplicación Práctica en la Industria de Harina de Pescado. Colegio de Ingenieros del Perú. Lima-Perú.

IMARPE/ITP. 1996. Compendio Biológico Tecnológico de las Principales Especies Hidrobiológicas Comerciales del Perú. Lima. Perú.

Ingram M., Bray D., Clark D., Dolman C., Elliot R., \& Thaetcher F. 1983. Microorganismos de los Alimentos. Técnicas de Análisis Microbiológicos. Vol. I. Segunda Edición. Ed. Acribia S. A., Zaragoza.

Jordán R. \& Chirinos A. 1965. La Anchoveta (Engraulis ringens). Conocimiento Actual sobre su Biología, Ecología, y Pesquería. IMARPE, Informe $N^{\circ}$ 6, Callao Perú.

Laevastu T. 1971. Manual de Métodos de Biología Pesquera. Ed. Acribia S.A. - FAO. Zaragoza 213 p.

Ludorff W. 1963. El Pescado y sus productos. Ed. Acribia S.A. Zaragoza.

Morales M., Faundez G. \& Figueroa G. 1999. Determinación de residuos de Cobre en Carne de Pollo y Cerdo Procesadas Experimentalmente sobre Superficies de este Metal. Unidad de Microbiología, INTA, Universidad de Chile.

Nagakura K. 1972. General Analysis in Utilization of Marine Products. Okada, M.; Hirao, S.; Noguchi, E.; Suzuki, T.; Yokoseki, M. (Editores). Overseas Technical Cooperation Agency, Tokio.

Pearson D. 1976. Técnicas de Laboratorio para el Análisis de Alimentos. Editorial Acribia S.A., Zaragoza.

Pesquera Capricornio S.A. 2003. Superintendencia de Planta-Callao.
Pizardi C. 1975. Estudio sobre la Fermentación Láctica del machete (Brevoortia maculata chilcae)" Tesis Ingeniero Pesquero, Universidad Nacional Agraria La Molina. Lima Perú.

Ramírez C. 1979. Procesamiento de la anchoveta (Engraulis ringens) sazonada en salsa de soya (Shoyu)”. Tesis Ingeniero Pesquero. Universidad Nacional Agraria La Molina. Lima Perú.

Ricque D., Cruz E., San Martín P. \& Pike I. 1996. Efecto de Aceites Oxidados y Deficiencia en Vitamina E y/o Antioxidante Artificial en Dietas para Camarón. Avances en Nutrición Acuícola III. Memorias del Tercer Simposium Internacional de Nutrición Acuícola. Universidad Autónoma de Nueva León, Monterrey México.

Rodríguez M. 2002. Recuperación de Proteínas del Agua de Cola Empleando Polímeros Naturales. Tesis Ingeniero Pesquero, Universidad Nacional Agraria La Molina. Lima Perú.

Román C. 1968. Conservación de la Anchoveta con Aditivos Químicos para un mejor Rendimiento en la obtención de Harina de Pescado. Tesis Ingeniero Pesquero, UNJFSC. Huacho - Lima.

Ruiter A. 1999. El Pescado y los Productos Derivados de la Pesca. Composición, Propiedades Nutritivas y Estabilidad. Editorial Acribia S.A. Zaragoza. 310 p

Sequi P. 2002. La nobleza desconocida. Instituto Sperimentale per la Nutrizione delle Piante. Roma.

Shirasaka R. \& Arakaki, J. 1975. Cálculos relacionados con el Procesamiento de la Harina de Pescado. Universidad Nacional Agraria La Molina. Lima Perú.

\footnotetext{
${ }^{1}$ Universidad Nacional Agraria La Molina. Av. La Molina s/n Apartado 12-056 Lima 12 Perú. Correos electrónicos: $\quad$ droldan@lamolina.edu.pe;

jjm@lamolina.edu.pe; $\quad$ marvash@yahoo.com;
} willyquevedotam@yahoo.com 